\title{
Method for the minimum cost maximum flow determining in fuzzy dynamic network with nonzero flow bounds
}

\author{
Alexander Bozhenyuk ${ }^{1}$ Evgeniya Gerasimenko $^{1}$ Igor Rozenberg $^{2}$ Irina Perfilieva $^{3}$ \\ ${ }^{1}$ Southern Federal University, Nekrasovsky 44, 347922 Taganrog, Russia \\ ${ }^{2}$ Public Corporation "Research and Development Institute of Railway Engineers", Nizhegorodskaya 27/1, 109029 \\ Moscow, Russia \\ ${ }^{3}$ Centre of Excellence IT4Innovations division of the University of Ostrava Institute for Research and Applications of \\ Fuzzy Modeling, 30. dubna 22, 70100 Ostrava 1, Czech Republic
}

\begin{abstract}
In this paper the method for the minimum cost flow determining in fuzzy dynamic network with nonzero lower flow bounds is proposed. Considered method takes into account fuzzy nature of networks' parameters and deals with fuzzy arc flow bounds, fuzzy transmission costs. Fuzzy arc flow bounds, costs and transit times can vary depending on the flow departure time. The method allows introducing the notion of "fuzzy network" instead of "stationary-dynamic" network, considered in conventional flow literature. The sphere of application for considered problem is presented.
\end{abstract}

Keywords: Fuzzy dynamic network, fuzzy minimum cost flow with nonzero lower flow bounds.

\section{Introduction}

Conventional tasks of maximum and minimum cost flow finding assume the instant flow, passing along the arcs of the graph [1]. Such tasks are called static flow tasks. In fact, it turns out that the flow spends certain time passing along the arcs of the graph. Then, we turn to dynamic networks, in which each flow unit passes from the source to the sink for a period of time less than given [2]. Dynamic network is a network $G=(X, A)$, where $X=\left\{x_{1}, x_{2} \ldots, x_{n}\right\}$ is the set of nodes, $A=\left\{\left(x_{i}, x_{j}\right)\right\}, i, j \in I=\overline{1, n}$ is the set of arcs. Two parameters are assigned to each arc of the dynamic graph $\left(x_{i}, x_{j}\right)$ : transit time $\tau_{i j}$ and arc capacity $u_{i j}$. The time horizon $T=\{0,1, \ldots, p\}$ determines that all flow units sent from the source must arrive in the sink within the time $p$ [3].

Dynamic networks describe complex systems, problems of decision-making, models, which parameters can vary over time [4]. Such models can be found in communication systems, economic planning, transportation systems and many other applications, so they have a wide practical application.

Fuzzy dynamic networks literature review is presented in [2]. Historically, the maximum flow finding in dynamic graphs was the first task in dynamic graphs, described in the literature. This problem is in finding of the maximum flow, passing from the source $(s)$ to the sink $(t), s, t \in X$ in the network for $p$ discrete time periods, starting from zero period of time.

The task of minimum cost flow finding in dynamic graphs is in searching of flows of the given value, which have minimum cost. This field, appeared later, is more complex sphere of investigations. Fleischer and Skutella [5] examined this problem. Cai et al. [6] considered networks with transit parameters. The sub problem of the minimum cost flow finding in dynamic graphs is the shortest path problem. This problem was widely reported in the literature by the authors Ahuja et al. [7] in terms of nonnegative transit times.

The fact that the flow, passing along the arcs of the graph, can have lower bounds usually isn't taken into account in the literature. For example, network that consists of railways, sea and air roads is considered. Therefore, the freight trains have certain level of load, which is more than profitability threshold, transport planes don't fly at a low load. Thus, it is necessary to introduce lower flow bounds, which can lead to the absence of feasible flow.

Despite the investigations of the authors in the field of dynamic graphs, the problems described above were not considered in the literature in fuzzy conditions. The authors Glockner et al. [8] considered the minimum cost flow problem in dynamic graphs as a task of linear programming. In fact, the flow bounds, the values of flows, passing along the arcs, transmission costs cannot be accurately measured according to their nature. The weather conditions, traffic jams, repairs influence flow bounds. Variations in petrol prices can influence transmission costs. Therefore, these parameters should be presented in a fuzzy form, such as fuzzy triangular numbers. Thus, we obtain a problem statement of the minimum cost maximum flow finding in the dynamic network in fuzzy conditions. The method of operating with fuzzy numbers is presented in [9].

In the present contribution we propose a method of the minimum cost maximum flow finding in fuzzy dynamic network. Presented method considers nonzero lower flow bounds, upper flow bounds, transmission costs in a fuzzy form and transit times depending on the departure time. The proposed method can be used for 
efficient cargo transportation. In the present paper it is demonstrated with GIS «ObjectLand».

The paper is structured as follows. The problem statement of the minimum cost maximum flow finding in fuzzy dynamic network with nonzero lower flow bounds and basic definitions and rules of proposed method are provided in the next section preliminaries. The third section deals with proposed method. The application of the proposed method is described in the fourth section. The last section is a conclusion and future work.

\section{Preliminaries}

This section is devoted to basic definitions and rules that underlie the proposed method and contains the description of the problem statement of the minimum cost maximum flow finding in fuzzy dynamic network with nonzero lower flow bounds.

\subsection{Basic definitions and problem statement}

\section{Definition 1}

Fuzzy stationary-dynamic network is a network which is represented by a fuzzy directed graph $\tilde{G}=(X, \tilde{A})$, where $X=\left\{x_{1}, x_{2}, \ldots, x_{n}\right\}$ is the set of nodes, $\tilde{A}=\left\{\mu_{\tilde{A}}\left\langle x_{i}, x_{j}\right\rangle /\left\langle x_{i}, x_{j}\right\rangle\right\}$,

$\left\langle x_{i}, x_{j}\right\rangle \in X^{2}, \mu_{\tilde{A}}\left\langle x_{i}, x_{j}\right\rangle-$ is the fuzzy set of arcs with the assigned membership function $\mu_{\bar{A}}\left\langle x_{i}, x_{j}\right\rangle$ of the directed arc $\left(x_{i}, x_{j}\right)$ to the set $\tilde{A}$. Two parameters are assigned to each arc of the dynamic graph $\left(x_{i}, x_{j}\right)$ : transit time $\tau_{i j}$ and arc capacity $u_{i j}$. The time horizon $T=\{0,1, \ldots, p\}$ determines that all flow units sent from the source must arrive in the sink before the time $p$.

\section{Definition 2}

Fuzzy dynamic network is a network which is represented by a fuzzy directed graph $\tilde{G}=(X, \tilde{A})$, where $X=\left\{x_{1}, x_{2}, \ldots, x_{n}\right\} \quad$ is the set of nodes, $\tilde{A}=\left\{\mu_{\tilde{A}}\left\langle x_{i}, x_{j}\right\rangle /\left\langle x_{i}, x_{j}\right\rangle\right\},\left\langle x_{i}, x_{j}\right\rangle \in X^{2}$,

$\mu_{\tilde{A}}\left\langle x_{i}, x_{j}\right\rangle-$ is the fuzzy set of arcs with the assigned membership function $\mu_{\tilde{A}}\left\langle x_{i}, x_{j}\right\rangle$ of the directed arc $\left(x_{i}, x_{j}\right)$ to the set $\tilde{A}$. Two parameters depending on the flow departure time are assigned to each arc of the dynamic graph $\left(x_{i}, x_{j}\right)$ : transit time $\tau_{i j}(\theta)$ and arc capacity $\tilde{u}_{i j}(\theta)$. The time horizon $T=\{0,1, \ldots, p\}$ determines that all flow units sent from the source must arrive in the sink before the time $p$.

The problem statement of the minimum cost maximum flow finding in fuzzy dynamic network with fuzzy lower and upper flow bounds is as follows:

$$
\text { Minimize } \sum_{\theta=0}^{p} \sum_{\left(x_{i}, x_{j}\right) \in \tilde{A}} \tilde{c}_{i j}(\theta) \times \tilde{\xi}_{i j}(\theta),
$$

$$
\begin{aligned}
& \sum_{\theta=0}^{p} \sum_{x_{j} \in X}\left[\tilde{\xi}_{s j}(\theta)-\tilde{\xi}_{j s}\left(\theta-\tau_{j s}(\theta)\right)\right]-\tilde{v}(p)=\tilde{0}, \\
& \sum_{x_{j} \in X}\left[\tilde{\xi}_{i j}(\theta)-\tilde{\xi}_{j i}\left(\theta-\tau_{j i}(\theta)\right)\right]=\tilde{0}, x_{i} \neq s, t ; \theta \in T, \\
& \sum_{\theta=0}^{p} \sum_{x_{j} \in X}\left[\tilde{\xi}_{t j}(\theta)-\tilde{\xi}_{j t}\left(\theta-\tau_{j t}(\theta)\right)\right]+\tilde{v}(p)=\tilde{0}, \\
& \tilde{l}_{i j}(\theta) \leq \tilde{\xi}_{i j}(\theta) \leq \tilde{u}_{i j}(\theta), \theta+\tau_{i j}(\theta) \leq p, \theta \in T .
\end{aligned}
$$

The equation (1) means that it is necessary to find the minimum cost maximum flow for the specified number of time periods, where The equation (2) indicates that the maximum flow value $\tilde{v}$ for $p$ time periods is equal to the total flow, leaving the source for $p$ time periods $\sum_{\theta=0}^{p} \tilde{\xi}_{s j}(\theta)$. The equation (4) reflects that the maximum flow value $\tilde{v}$ for $p$ time periods is equal to the total flow entering the sink for $p$ time periods $\sum_{\theta=0}^{p} \tilde{\xi}_{j t}\left(\theta-\tau_{j t}\right)$. The flow entering the source $\sum_{\theta=0}^{p} \tilde{\xi}_{j s}\left(\theta-\tau_{j s}\right)$ for $p$ time periods is equal to the flow $\sum_{\theta=0}^{p} \tilde{\xi}_{t j}(\theta)$ leaving the sink for $p$ time periods and is equal to $\tilde{0}$. For each node $x_{i}$ except the source and the sink and for each time period $\theta$ the amount of flow $\tilde{\xi}_{j i}\left(\theta-\tau_{j i}\right)$ entering $x_{i}$ at each period of time $\left(\theta-\tau_{j i}\right)$ is equal to the amount of flow $\tilde{\xi}_{i j}(\theta)$ leaving $x_{i}$ at time $\theta$ as stated in (3). The inequality (5) indicates that the flows $\tilde{\xi}_{i j}(\theta)$ for time periods $\theta: \theta+\tau_{i j}(\theta) \leq p, \theta \in T$ should be more than lower flow bounds $\tilde{l}_{i j}(\theta)$ and less than upper flow bounds $\tilde{u}_{i j}(\theta)$ along the corresponding arcs.

By the other words it is necessary to transport maximum flow units $\tilde{v}(p)$ of the minimum cost in dynamic network taking into account lower flow bounds in such a way that the last flow unit would enter the sink at time period not later than $p$. In this case, upper flow bounds, lower flow bounds and transmission costs are transit.

\subsection{Basic rules}

Presented method of the minimum cost maximum flow determining considers transforming to the timeexpanded fuzzy graph corresponding to the initial graph (rule 1) defined in [10].

Rule 1 of the time-expanded fuzzy static graph $\tilde{G}_{p}$ construction from the given fuzzy dynamic graph $\tilde{G}$ for the minimum cost maximum flow finding in fuzzy $d y$ - 
namic network with fuzzy transit nonzero lower flow bounds, upper flow bounds and costs

Let $\tilde{G}_{p}=\left(X_{p}, \tilde{A}_{p}\right)$ represent fuzzy time-expanded static graph of the original dynamic fuzzy graph. The set of nodes $X_{p}$ of the graph $\tilde{G}_{p}$ is defined as $X_{p}=\left\{\left(x_{i}, \theta\right):\left(x_{i}, \theta\right) \in X \times T\right\}$. The set of arcs $\tilde{A}_{p}$ consists of arcs going from each node-time pair $\left(x_{i}, \theta\right) \in X_{p}$ to every node-time pair $\left(x_{j}, \theta+\tau_{i j}(\theta)\right)$, where $x_{j} \in \Gamma\left(x_{i}\right)$ and $\theta+\tau_{i j}(\theta) \leq p$. Fuzzy upper flow bounds $\tilde{u}\left(x_{i}, x_{j}, \theta, \theta+\tau_{i j}(\theta)\right)$ connecting $\left(x_{i}, \theta\right)$ with $\left(x_{j}, \theta+\tau_{i j}(\theta)\right)$ are equal to $\tilde{u}_{i j}(\theta)$. Fuzzy lower flow bounds $\tilde{l}\left(x_{i}, x_{j}, \theta, \theta+\tau_{i j}(\theta)\right)$ joining $\left(x_{i}, \theta\right)$ with $\left(x_{j}, \theta+\tau_{i j}(\theta)\right)$ are equal to $\tilde{l}_{i j}(\theta)$, transmission cost $\tilde{c}\left(x_{i}, x_{j}, \theta, \theta+\tau_{i j}(\theta)\right)$ of one flow unit along the arc connecting the node-time pair $\left(x_{i}, \theta\right)$ with $\left(x_{j}, \theta+\tau_{i j}(\theta)\right)$ is equal to $\tilde{c}_{i j}(\theta)$.

After that turn to fuzzy time-expanded graph without lower flow bounds according to the rule 2 provided in [10].

Rule 2 of transformation from the time-expanded fuzzy graph to fuzzy graph without lower flower bounds for the minimum cost maximum flow finding in fuzzy dynamic network with fuzzy transit nonzero lower flow bounds, upper flow bounds and costs

Turn to the time-expanded graph $\tilde{G}_{p}^{*}=\left(X_{p}^{*}, \tilde{A}_{p}^{*}\right)$ without lower flow bounds from initial fuzzy time-expanded graph $\tilde{G}_{p}=\left(X_{p}, \tilde{A}_{p}\right)$ introducing the artificial source $s^{*}$ and sink $t^{*}$ and arcs, connecting the node-time pair ( $t, \forall \theta \in \mathrm{T})$ and $(s, \forall \theta \in \mathrm{T})$ with upper fuzzy flow bound $\tilde{u}^{*}(t, s, \forall \theta \in T, \forall \theta \in T)=\infty$, lower fuzzy flow bound $\tilde{l}^{*}(t, s, \forall \theta \in T, \forall \theta \in T)=\tilde{0}$, transmission cost $\tilde{c}^{*}(t, s, \forall \theta \in T, \forall \theta \in T)=\tilde{0}$. It means that every node $t$ in each time period from $p$ is connected with every node $s$ at all time periods in the graph $\tilde{G}_{p}^{*}$. For each arc connecting the node-time pair $\left(x_{i}, \vartheta\right)$ with the nodetime pair $\left(x_{j}, \theta=\vartheta+\tau_{i j}(\vartheta)\right)$ with nonzero lower fuzzy flow bound $\tilde{l}\left(x_{i}, x_{j}, \vartheta, \theta\right) \neq \tilde{0}$ introduce the following modification $\tilde{l}\left(x_{i}, x_{j}, \vartheta, \theta\right) \neq \tilde{0}$ :

1) reduce $\tilde{u}\left(x_{i}, x_{j}, \vartheta, \theta\right)$ to $\tilde{u}^{*}\left(x_{i}, x_{j}, \vartheta, \theta\right)=\tilde{u}\left(x_{i}, x_{j}, \vartheta, \theta\right)-\tilde{l}\left(x_{i}, x_{j}, \vartheta, \theta\right)$, $\tilde{l}\left(x_{i}, x_{j}, \vartheta, \theta\right)$ to $\tilde{0}, \tilde{c}^{*}\left(x_{i}, x_{j}, \vartheta, \theta\right)=\tilde{c}\left(x_{i}, x_{j}, \vartheta, \theta\right)$.

2) Introduce the arcs connecting $s^{*}$ with $\left(x_{j}, \theta\right)$ and $t^{*}$ with $\left(x_{i}, \vartheta\right)$ with upper fuzzy flow bounds equal to lower fuzzy flow bounds $\tilde{u}^{*}\left(s^{*}, x_{j}, \forall, \vartheta\right)=\tilde{u}^{*}\left(x_{i}, t, \theta, \forall\right)=\tilde{l}\left(x_{i}, x_{j}, \theta, \vartheta\right) \quad$ lower fuzzy flow bounds $\tilde{l}^{*}\left(s^{*}, x_{j}, \forall, \vartheta\right)=\tilde{l}^{*}\left(x_{i}, t, \theta, \forall\right)=\tilde{0}$ and transmission

costs $\tilde{c}^{*}\left(s^{*}, x_{j}, \forall, \vartheta\right)=\tilde{c}^{*}\left(x_{i}, t, \theta, \forall\right)=\tilde{0}$

Introduce the modified definition of the fuzzy residual network of the time-expanded graph for the minimum cost dynamic flow finding with nonzero lower flow bounds, mentioned in [10]. The criteria of obtaining feasible flow in the time-expanded fuzzy graph is the maximum flow of the minimum cost determining in the modified graph without lower flow bounds.

\section{Definition 3}

Fuzzy residual network $\tilde{G}_{p}^{* \mu}=\left(X_{p}^{* \mu}, \tilde{A}_{p}^{* \mu}\right)$ of the timeexpanded fuzzy graph for the minimum cost maximum flow finding in the dynamic network with fuzzy nonzero lower flower bounds, upper flow bounds and transmission costs is the network constructed in such a way: if the flow $\tilde{\xi}^{*}\left(x_{i}, x_{j}, \theta, \vartheta\right)$ going from the nodetime pair $\left(x_{i}^{*}, \theta\right)$ to the node-time pair $\left(x_{j}^{*}, \vartheta\right)$ is less than appropriate arc capacity $\tilde{u}^{*}\left(x_{i}, x_{j}, \theta, \vartheta\right)$, i.e. $\tilde{\xi}^{*}\left(x_{i}, x_{j}, \theta, \vartheta\right)<\tilde{u}^{*}\left(x_{i}, x_{j}, \theta, \vartheta\right)$ in $\tilde{G}_{p}^{*}$, then include appropriate arc, connecting the node-time pair $\left(x_{i}^{* \mu}, \theta\right)$ with the node-time pair $\left(x_{j}^{* \mu}, \vartheta\right)$ in $\tilde{G}_{p}^{* \mu}$ with arc capacity $\tilde{u}^{* \mu}\left(x_{i}, x_{j}, \theta, \vartheta\right)=\tilde{u}^{*}\left(x_{i}, x_{j}, \theta, \vartheta\right)-\tilde{\xi}^{*}\left(x_{i}, x_{j}, \theta, \vartheta\right)$, transit time $\tau^{* \mu}\left(x_{i}, x_{j}, \theta, \vartheta\right)=\tau^{*}\left(x_{i}, x_{j}, \theta, \vartheta\right), \quad$ transmission $\operatorname{cost} \tilde{c}^{* \mu}\left(x_{i}, x_{j}, \theta, \vartheta\right)=\tilde{c}^{*}\left(x_{i}, x_{j}, \theta, \vartheta\right)$. If the flow $\tilde{\xi}^{*}\left(x_{i}, x_{j}, \theta, \vartheta\right)$ going from the node-time pair $\left(x_{i}^{*}, \theta\right)$ to the node-time pair $\left(x_{j}^{*}, \vartheta\right)$ exceeds $\tilde{0}$, then include appropriate arc, connecting the node-time pair $\left(x_{j}{ }^{* \mu}, \vartheta\right)$ with the node-time pair $\left(x_{i}^{* \mu}, \theta\right)$ in $\tilde{G}_{p}^{* \mu}$ with arc capacity $\tilde{u}^{* \mu}\left(x_{j}, x_{i}, \vartheta, \theta\right)=\tilde{\xi}^{*}\left(x_{i}, x_{j}, \theta, \vartheta\right)$, transit time $\tau^{* \mu}\left(x_{j}, x_{i}, \vartheta, \theta\right)=-\tau^{*}\left(x_{i}, x_{j}, \theta, \vartheta\right), \quad$ transmission cost $\tilde{c}^{* \mu}\left(x_{j}, x_{i}, \vartheta, \theta\right)=-\tilde{c}^{*}\left(x_{i}, x_{j}, \theta, \vartheta\right)$.

If the feasible flow is found in the time-expanded fuzzy graph, transform it by finding the maximum flow of the minimum cost according to the rule $\mathbf{3}$, given in [10].

Rule 3 of fuzzy residual network construction with feasible flow for the minimum cost maximum flow finding in fuzzy dynamic network with transit nonzero lower flow bounds, upper flow bound and transmission costs If the flow $\tilde{\xi}\left(x_{i}, x_{j}, \theta, \vartheta\right)$, going from the node-time pair $\left(x_{i}, \theta\right)$ to the node-time pair $\left(x_{j}, \vartheta\right)$ is less than appropriate arc capacity $\tilde{u}\left(x_{i}, x_{j}, \theta, \vartheta\right)$, i.e. $\tilde{\xi}\left(x_{i}, x_{j}, \theta, \vartheta\right)<\tilde{u}\left(x_{i}, x_{j}, \theta, \vartheta\right)$ in $\tilde{G}_{p}$, then include appropriate arc, connecting the node-time pair $\left(x_{i}^{\mu}, \theta\right)$ with the node-time pair $\left(x_{j}^{\mu}, \vartheta\right)$ in $\tilde{G}_{p}^{\mu}(\tilde{\xi})$ with arc capacity $\quad \tilde{u}^{\mu}\left(x_{i}, x_{j}, \theta, \vartheta\right)=\tilde{u}\left(x_{i}, x_{j}, \theta, \vartheta\right)-\tilde{\xi}\left(x_{i}, x_{j}, \theta, \vartheta\right)$, transit time $\tau^{\mu}\left(x_{i}, x_{j}, \theta, \vartheta\right)=\tau\left(x_{i}, x_{j}, \theta, \vartheta\right)$, transmis- 
sion $\operatorname{cost} \tilde{c}^{\mu}\left(x_{i}, x_{j}, \theta, \vartheta\right)=\tilde{c}\left(x_{i}, x_{j}, \theta, \vartheta\right)$. If the flow $\tilde{\xi}\left(x_{i}, x_{j}, \theta, \vartheta\right)$, connecting the node-time pair $\left(x_{i}, \theta\right)$ with $\left(x_{j}, \vartheta\right)$ exceeds $\tilde{l}\left(x_{i}, x_{j}, \theta, \vartheta\right)$ in $\tilde{G}_{p}$, i.e. $\tilde{\xi}\left(x_{i}, x_{j}, \theta, \vartheta\right)>\tilde{l}\left(x_{i}, x_{j}, \theta, \vartheta\right)$, then include appropriate arc, connecting the node-time pair $\left(x_{j}^{\mu}, \vartheta\right)$ with the node-time pair $\left(x_{i}^{\mu}, \theta\right)$ in $\tilde{G}_{p}^{\mu}(\tilde{\xi})$ with arc capacity $\tilde{u}^{\mu}\left(x_{j}, x_{i}, \vartheta, \theta\right)=\tilde{\xi}\left(x_{i}, x_{j}, \theta, \vartheta\right)-\tilde{l}\left(x_{i}, x_{j}, \theta, \vartheta\right), \quad$ transit time $\tau^{\mu}\left(x_{j}, x_{i}, \vartheta, \theta\right)=-\tau\left(x_{i}, x_{j}, \theta, \vartheta\right), \quad$ transmission $\operatorname{cost} \tilde{c}^{\mu}\left(x_{j}, x_{i}, \vartheta, \theta\right)=-\tilde{c}\left(x_{i}, x_{j}, \theta, \vartheta\right)$.

\section{Proposed method}

We represent the formal algorithm describing the solution of the minimum cost maximum dynamic flow finding problem with upper and lower fuzzy flow bounds in a fuzzy transportation network with time-varying fuzzy flow bounds, transmission costs and time-varying crisp flow transit times along the arcs. Computational complexity of the proposed method is based on the computational complexity of the augmenting minimum cost path finding method of the Ford and Bellman, which searches the shortest path from the one node to others within the time $O(X A)$.

Step 1. Go to the time-expanded fuzzy static graph $\tilde{G}_{p}$ from the given fuzzy dynamic graph $\tilde{G}$ by expanding the original dynamic graph in the time dimension by making a separate copy of every node $x_{i} \in X$ at every time $\theta \in T$ according to the rule $\mathbf{1}$.

Step 2. Determine, if the time-expanded fuzzy graph $\tilde{G}_{p}$, corresponded to the initial dynamic graph $\tilde{G}$, has a feasible flow. Turn to the fuzzy graph $\tilde{G}_{p}^{*}$ according to the rule 2 .

Step 3. Build a fuzzy residual network $\tilde{G}_{p}^{* \mu}$ by the fuzzy network $\tilde{G}_{p}^{*}$ according to the definition 3 .

Step 4. Search the augmenting minimum cost path $\tilde{P}_{p}^{* \mu}$ from the artificial source $s^{*}$ to the artificial sink $t^{*}$ in the $\tilde{G}_{p}^{* \mu}$ according to the Ford's algorithm.

4.1. Go to the step 5 if the augmenting path $\tilde{P}_{p}^{* \mu}$ is found.

4.2. If the path is failed to find, the flow value $\tilde{\phi}^{*}<\sum_{\tilde{l}\left(x_{i}, x_{j}, \vartheta, \theta\right) \neq \tilde{0}} \tilde{l}\left(x_{i}, x_{j}, \vartheta, \theta\right)$ is obtained, which is the maximum flow in $\tilde{G}_{p}^{*}$. It means that it is impossible to pass any unit of flow, but not all the artificial arcs are saturated. Therefore, the time-expanded graph $\tilde{G}_{p}$ has no feasible flow as the initial dynamic fuzzy graph $\tilde{G}$ and the task has no solution. Exit.
Step 5. Pass $\tilde{\delta}_{p}^{* \mu}=\min \left[\tilde{u}\left(\tilde{P}_{p}^{* \mu}\right)\right]$, $\tilde{u}\left(\tilde{P}_{p}^{* \mu}\right)=\min \left[\tilde{u}^{* \mu}\left(x_{i}, x_{j}, \theta, \vartheta\right)\right],\left(x_{i}, \theta\right),\left(x_{j}, \vartheta\right) \in \tilde{P}_{p}^{* \mu}$ flow units along the minimum cost path $\tilde{P}_{p}^{* \mu}$.

Step 6. Update the fuzzy flow values in the graph $\tilde{G}_{p}^{*}$ : replace the fuzzy flow $\tilde{\xi}^{*}\left(x_{j}, x_{i}, \vartheta, \theta\right)$ along the corresponding arcs going from $\left(x_{j}^{*}, \theta\right)$ to $\left(x_{i}^{*}, \vartheta\right)$ from $\tilde{G}_{p}^{*}$ by $\tilde{\xi}^{*}\left(x_{j}, x_{i}, \vartheta, \theta\right)-\tilde{\delta}_{p}^{* \mu}$ for arcs connecting nodetime pair $\left(x_{i}^{* \mu}, \vartheta\right)$ with $\left(x_{j}^{* \mu}, \theta\right)$ in $\tilde{G}_{p}^{* \mu}$ such as $\left(\left(x_{i}^{* \mu}, \vartheta\right),\left(x_{j}^{* \mu}, \theta\right)\right) \notin \tilde{A}_{p}^{*}, \quad\left(\left(x_{i}^{* \mu}, \vartheta\right),\left(x_{j}^{* \mu}, \theta\right)\right) \in \tilde{A}_{p}^{* \mu} \quad$ and replace the fuzzy flow $\tilde{\xi}^{*}\left(x_{i}, x_{j}, \vartheta, \theta\right)$ along the arcs going from $\left(x_{i}^{*}, \theta\right)$ to $\left(x_{j}^{*}, \vartheta\right)$ from $\tilde{G}_{p}^{*}$ by $\tilde{\xi}^{*}\left(x_{i}, x_{j}, \vartheta, \theta\right)+\tilde{\delta}_{p}^{* \mu}$ for arcs connecting node-time pair $\left(x_{i}^{* \mu}, \theta\right) \quad$ with $\quad\left(x_{j}^{* \mu}, \vartheta\right)$ in $\tilde{G}_{p}^{* \mu}$ such as $\left(\left(x_{i}^{* \mu}, \vartheta\right),\left(x_{j}^{* \mu}, \theta\right)\right) \in \tilde{A}_{p}^{*}, \quad\left(\left(x_{i}^{* \mu}, \vartheta\right),\left(x_{j}^{* \mu}, \theta\right)\right) \in \tilde{A}_{p}^{* \mu}$. Replace $\tilde{\xi}^{*}\left(x_{i}, x_{j}, \vartheta, \theta\right)$ by $\tilde{\xi}^{*}\left(x_{i}, x_{j}, \vartheta, \theta\right)+\tilde{\delta}_{p}^{* \mu} \times \tilde{P}_{p}^{* \mu}$.

Step 7. Compare flow value $\tilde{\xi}^{*}\left(x_{i}, x_{j}, \theta, \vartheta\right)+\tilde{\delta}_{p}^{* \mu} \times \tilde{P}_{p}^{* \mu}$ and the sum of the lower flow bounds $\sum_{i\left(x_{i}, x_{j}, \theta, \vartheta\right) \neq \overline{0}} \tilde{l}\left(x_{i}, x_{j}, \theta, \vartheta\right)$ :

7.1. If the flow value $\tilde{\xi}^{*}\left(x_{i}, x_{j}, \vartheta, \theta\right)+\tilde{\delta}_{p}^{* \mu} \times \tilde{P}_{p}^{* \mu}$ of the minimum cost $\tilde{c}\left(\tilde{\xi}^{*}\left(x_{i}, x_{j}, \vartheta, \theta\right)+\tilde{\delta}_{p}^{* \mu} \times P_{p}^{* \mu}\right)$ is less than $\sum_{\tilde{l}\left(x_{i}, x_{j}, \vartheta, \theta\right) \neq \tilde{0}} \tilde{l}\left(x_{i}, x_{j}, \vartheta, \theta\right)$ and is less than given flow value $\tilde{\sigma}^{*}<\sum_{i\left(x_{i}, x_{j}, \theta, \vartheta\right) \neq \tilde{0}} \tilde{l}\left(x_{i}, x_{j}, \theta, \vartheta\right)$, i.e. not all artificial arcs become saturated, go to the step 3 .

7.2. If the flow value $\tilde{\xi}^{*}\left(x_{i}, x_{j}, \vartheta, \theta\right)+\tilde{\delta}_{p}^{* \mu} \times \tilde{P}_{p}^{* \mu}$ of the minimum cost $\tilde{c}\left(\tilde{\xi}^{*}\left(x_{i}, x_{j}, \vartheta, \theta\right)+\tilde{\delta}_{p}^{* \mu} \times P_{p}^{* \mu}\right)$ is $\tilde{\sigma}^{*}=\sum_{i\left(x_{i}, x_{j}, \theta, \vartheta\right) \neq \tilde{0}} \tilde{l}\left(x_{i}, x_{j}, \theta, \vartheta\right)$ i.e. all arcs from the artificial source to the artificial sink become saturated, then the value $\tilde{\xi}^{*}\left(x_{i}, x_{j}, \vartheta, \theta\right)+\tilde{\delta}_{p}^{* \mu} \times \tilde{P}_{p}^{* \mu}$ is required value of maximum flow $\tilde{\sigma}^{*}$ of the minimum cost $\tilde{c}\left(\tilde{\xi}^{*}\left(x_{i}, x_{j}, \vartheta, \theta\right)+\tilde{\delta}_{p}^{* \mu} \times P_{p}^{* \mu}\right)$. In this case the total flow along the artificial arcs connecting the node-time pairs $(t, \forall \theta \in \mathrm{T})$ with $(s, \forall \theta \in \mathrm{T})$, which is equal to $\sum_{\theta=0}^{p} \tilde{\xi}^{*}(t, s, \forall \theta \in T, \forall \theta \in T)$ in $\tilde{G}_{p}^{*}$ determines the feasible flow in time-expanded graph $\tilde{G}_{p}$ with the flow value $\sum_{\theta=0}^{p} \tilde{\xi}^{*}(t, s, \forall \theta \in T, \forall \theta \in T)=\tilde{\sigma} \quad$ of minimum cost. Turn to the graph $\tilde{G}_{p}$ from the graph $\tilde{G}_{p}^{*}$. The network $\tilde{G}(\tilde{\xi})$ is obtained. Go to the step 8. 
Step 8. Construct the residual network $\tilde{G}_{p}^{\mu}(\tilde{\xi})$ taking into account the feasible flow vector $\tilde{\xi}=\left(\tilde{\xi}\left(x_{i}, x_{j}, \vartheta, \theta\right)\right)$ in $\tilde{G}_{p}(\tilde{\xi})$ adding the artificial source and sink and the arcs with infinite arc capacity and zero cost, connecting $s^{\prime}$ with true sources and $t$ with true sinks according to the rule 3 .

Step 9. Define the minimum cost path $\tilde{P}_{p}^{\mu}$ according to the Ford's algorithm from $s^{\prime}$ to $t^{\prime}$ in the constructed residual network $\tilde{G}_{p}^{\mu}(\tilde{\xi})$.

9.1. If the path $\tilde{P}_{p}^{\mu}$ is found, go to the step $\mathbf{1 0}$.

9.2. If the path doesn't exist, therefore the maximum flow value $\tilde{\xi}\left(x_{i}, x_{j}, \theta, \vartheta\right)+\tilde{\delta}_{p}^{\mu} \times \tilde{P}_{p}^{\mu}=\tilde{v}(p)$ of the minimum cost $\tilde{c}\left(\tilde{\xi}\left(x_{i}, x_{j}, \theta, \vartheta\right)+\tilde{\delta}_{p}^{\mu} \times \tilde{P}_{p}^{\mu}\right)$ is found in the time-expanded fuzzy graph. Go to the step 12.

Step 10. Pass the flow value $\tilde{\delta}_{p}^{\mu}=\min \left[\tilde{u}\left(\tilde{P}_{p}^{\mu}\right)\right], \tilde{u}\left(\tilde{P}_{p}^{\mu}\right)=\min \left[\tilde{u}^{\mu}\left(x_{i}, x_{j}, \theta, \vartheta\right)\right]$, $\left(x_{i}, \theta\right),\left(x_{j}, \vartheta\right) \in \tilde{P}_{p}^{\mu}$ along the found path.

Step 11. Update the flow values in the graph $\tilde{G}_{p}(\tilde{\xi})$ : replace the flow $\tilde{\xi}\left(x_{j}, x_{i}, \theta, \vartheta\right)$ by $\tilde{\xi}\left(x_{j}, x_{i}, \vartheta, \theta\right)-\tilde{\delta}_{p}^{\mu}$ along the corresponding arcs, going from $\left(x_{j}, \theta\right)$ to $\left(x_{i}, \vartheta\right)$ from $\tilde{G}_{p}(\tilde{\xi})$ for arcs, connecting node-time pair $\left(x_{i}^{\mu}, \vartheta\right) \quad$ with $\left(x_{j}^{\mu}, \theta\right)$ in $\tilde{G}_{p}^{\mu}(\tilde{\xi})$ such as $\left(\left(x_{i}^{\mu}, \vartheta\right),\left(x_{j}^{\mu}, \theta\right)\right) \notin \tilde{A}_{p},\left(\left(x_{i}^{\mu}, \vartheta\right),\left(x_{j}^{\mu}, \theta\right)\right) \in \tilde{A}_{p}^{\mu} \quad$ and replace the flow $\tilde{\xi}\left(x_{i}, x_{j}, \vartheta, \theta\right)$ by $\tilde{\xi}\left(x_{i}, x_{j}, \vartheta, \theta\right)+\tilde{\delta}_{p}^{\mu}$ along the corresponding arcs, going from $\left(x_{i}, \theta\right)$ to $\left(x_{j}, \vartheta\right)$ from $\tilde{G}_{p}(\tilde{\xi})$ for arcs, connecting node-time pair $\left(x_{i}^{\mu}, \theta\right)$ with $\left(x_{j}^{\mu}, \vartheta\right)$ in $\tilde{G}_{p}^{\mu}(\tilde{\xi})$ such as $\left(\left(x_{i}^{\mu}, \theta\right),\left(x_{j}^{\mu}, \vartheta\right)\right) \in \tilde{A}_{p},\left(\left(x_{i}^{\mu}, \theta\right),\left(x_{j}^{\mu}, \vartheta\right)\right) \in \tilde{A}_{p}^{\mu}$. Replace the flow value $\tilde{\xi}\left(x_{i}, x_{j}, \vartheta, \theta\right) \rightarrow \tilde{\xi}\left(x_{i}, x_{j}, \vartheta, \theta\right)+\tilde{\delta}_{p}^{\mu} \times \tilde{P}_{p}^{\mu}$ and go to the step 8.

Step 12. Turn to the initial dynamic graph $\tilde{G}$ from the time-expanded static graph $\tilde{G}_{p}$ as follows: the given dynamic flow of the minimum cost in the graph $\tilde{G}$ for $p$ time periods is equal to the flow, leaving the set of sources for all time periods and entering the set of sinks for all time periods before $p$. Each path, connecting the node-time pairs $(s, \vartheta)$ with $\left(t, \varsigma=\vartheta+\tau_{s t}(\vartheta)\right), \zeta \in T$, with the flow $\tilde{\xi}(s, t, \vartheta, \varsigma)$ of the cost $\tilde{c}(\tilde{\xi}(s, t, \vartheta, \varsigma))$ in $\tilde{G}_{p}$ corresponds to the flow $\tilde{\xi}_{s t}(\vartheta)$ of the cost $\tilde{c}\left(\tilde{\xi}_{s t}(\vartheta)\right)$ in $\tilde{G}$.

The following theorems and consequences are valid.

\section{Theorem 1}

If the maximum flow of the minimum cost in the graph $\tilde{G}_{p}^{*}$ is equal to the sum of lower flow bounds $\tilde{\sigma}^{*}=\sum_{l_{i j} \neq 0} \tilde{l}_{i j}$, then the feasible flow of the value $\sum_{\theta=0}^{p} \tilde{\xi}^{*}(t, s, \forall \theta \in T, \forall \theta \in T)=\tilde{\sigma}$ exists in the graph $\tilde{G}_{p}$.

\section{Consequence 1.1}

If $\tilde{\sigma}^{*}=\sum_{l_{i j} \neq 0} \tilde{l}_{i j}$, then minimum cost flow vector $\tilde{\xi}=\left(\tilde{\xi}_{i j}\right)$ of the value $\tilde{\sigma}$ is defined as $\tilde{\xi}_{i j}=\tilde{\xi}_{i j}^{*}+\tilde{l}_{i j}$ is the feasible flow of the minimum cost in the graph $\tilde{G}_{p}$ of the value $\sum_{\theta=0}^{p} \tilde{\xi}^{*}(t, s, \forall \theta \in T, \forall \theta \in T)=\tilde{\sigma}$

If the maximum flow in the graph $\tilde{G}_{p}^{*}$ is less than sum of the lower flow bounds of the initial graph, then the feasible flow doesn't exist in the graph $\tilde{G}_{p}$, that is mentioned in the theorem 2.

\section{Theorem 2}

If the maximum flow $\tilde{\sigma}^{*}$ in the graph $\tilde{G}_{p}^{*}$ is less than sum of the lower flow bounds of the initial graph, i.e. $\tilde{\sigma}^{*}<\sum_{i\left(x_{i}, x_{j}, \theta, \vartheta\right) \neq 0} \tilde{l}\left(x_{i}, x_{j}, \theta, \vartheta\right)$, then feasible flow doesn't exist in the graph $\tilde{G}_{p}$.

\section{Practical implementation of the proposed meth- od results}

The results of the proposed method can be implemented for solving the routing problems and defining the transportation profitability on the railway networks. Practical implementation of the proposed method is realized by the railway map of the Russian Federation. The data for the program are taken from the GIS «Object Land» 2.6 $[11,12]$. The main sources of the input data are already constructed tables from GIS «Object Land» describing the railway network of Russia.

Necessary condition is possibility to use different ways of defining fuzzy numbers:

- Triangular fuzzy numbers.

- Trapezoidal fuzzy numbers.

- Interval fuzzy numbers.

Presented method assumes operating with centers of fuzzy numbers. The «blurring» of the resulting number is implemented in the end of the algorithm according to given basic values of the costs presented in the Fig. 1-3.

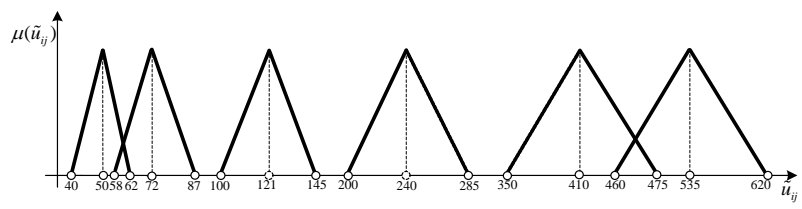

Fig. 1: Basic values of transmission costs. 


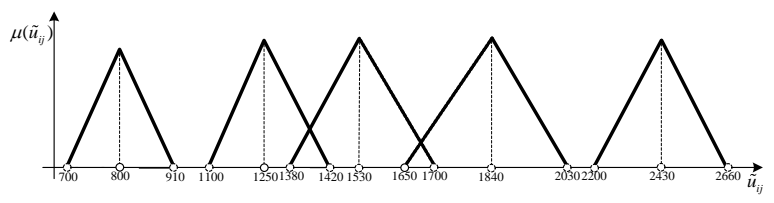

Fig. 2: Basic values of transmission costs.

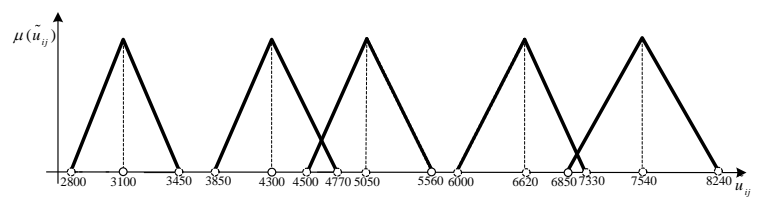

Fig. 3: Basic values of transmission costs.

Example data describing the plot of the railway network of the Rostov region is given in the Table 1.

\begin{tabular}{|c|c|c|c|c|c|}
\hline $\begin{array}{c}\text { Initial } \\
\text { sta- } \\
\text { tion } \\
\text { ID }\end{array}$ & $\begin{array}{c}\text { Ter- } \\
\text { minal } \\
\text { sta- } \\
\text { tion } \\
\text { ID }\end{array}$ & $\begin{array}{c}\text { Upper } \\
\text { fuzzy } \\
\text { flow } \\
\text { bound }\end{array}$ & $\begin{array}{c}\text { Low- } \\
\text { er } \\
\text { fuzzy } \\
\text { flow } \\
\text { bound }\end{array}$ & $\begin{array}{c}\text { Trans } \\
\text { mis- } \\
\text { sion } \\
\text { cost }\end{array}$ & $\begin{array}{c}\text { Trans } \\
\text { it } \\
\text { time }\end{array}$ \\
\hline 3352 & 3349 & 30,24, & $0,0,0$, & 30,25, & $4,4,1$, \\
& & 40,30 & 0 & 50,30 & 2 \\
1129 & 3428 & 25,18, & $0,0,0$, & 25,30, & $5,1,2$, \\
& & 20,20 & 0 & 80,20 & 3 \\
3350 & 1129 & 30,30, & $0,0,0$, & 30, & $4,1,1$, \\
& & 25,25 & 0 & 100, & 1 \\
3427 & 3352 & 10,18, & 0,10, & 30,60, & $5,1,3$, \\
& & 8,10 & 0,0 & 60,30 & 2 \\
3427 & 3428 & 10,15, & $0,0,0$, & 75,50, & $1,3,2$, \\
& & 18,18 & 0 & 18,18 & 1 \\
3427 & 1129 & 20,20, & 18,0, & 70,30, & $1,3,3$, \\
& & 25,18 & 0,0 & 20,18 & 3 \\
3349 & 3350 & 30,30, & $0,0,0$, & 30,30, & $4,4,1$, \\
& & 45,25 & 0 & 80,25 & 1 \\
\hline
\end{tabular}

Table 1: The example of the input data.

Thus, the input data can be any CSV-file of the given structure. The names of the particular fields are configurable parameters. The output data of the program are:

- $\quad$ Calculated maximum flow value of the minimum cost in fuzzy form.

- Flow distribution along the arcs of the network in the form of paths and flows along them.

The input data for the program are created by selection of the investigated area and exporting database records connected with this area, as shown in the Fig. 4.

Let us represent the sample data from the GIS ObjectLand in the form of the fuzzy network as shown in the Fig. 5.

Construct the time-expanded graph $\tilde{G}_{p}$. from the initial graph $\tilde{G}$, as shown in the Fig. 6 .

Construct the fuzzy time-expanded graph without lower flow bounds $\tilde{G}_{p}^{*}$ with artificial source and sink, as shown in the Fig. 7.

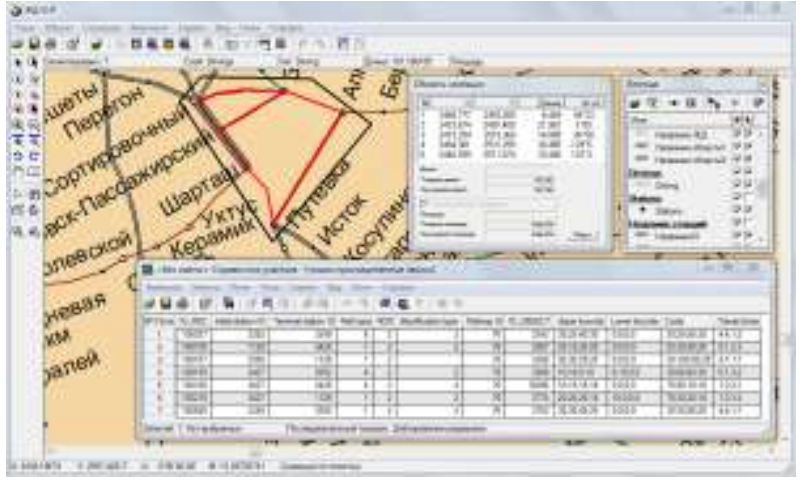

Fig. 4: The sample data based on the selection of the «plot» type objects.

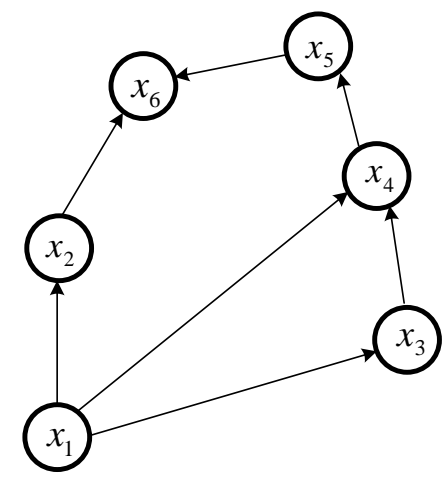

Fig. 5: Initial dynamic graph $\tilde{G}$.

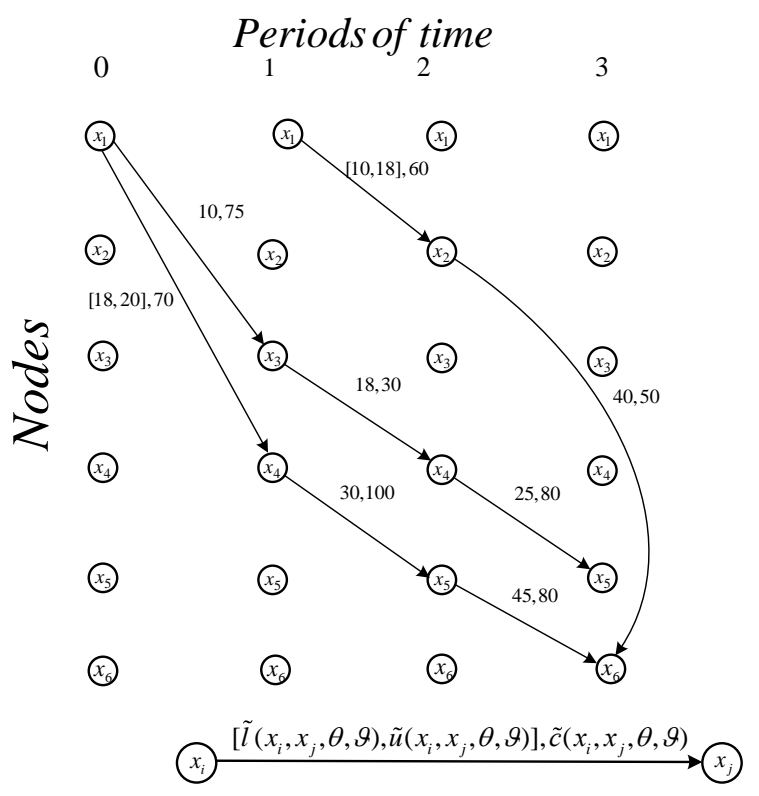

Fig. 6: The time-expanded graph $\tilde{G}_{p}$.

Build the fuzzy residual network $\tilde{G}_{p}^{* \mu}$ of the graph $\tilde{G}_{p}^{*}$ with 10 flow units (Fig. 8).

Consequentially building the paths of the minimum cost and pushing flows along them we obtain the maximum flow of the minimum cost (see Fig. 9) of the value 38 units. 
The minimal transmission costs of the 38 flow units is: $20 \cdot 70+18 \cdot 60+18 \cdot 50+20 \cdot 100+20 \cdot 80=6980$ conventional units.

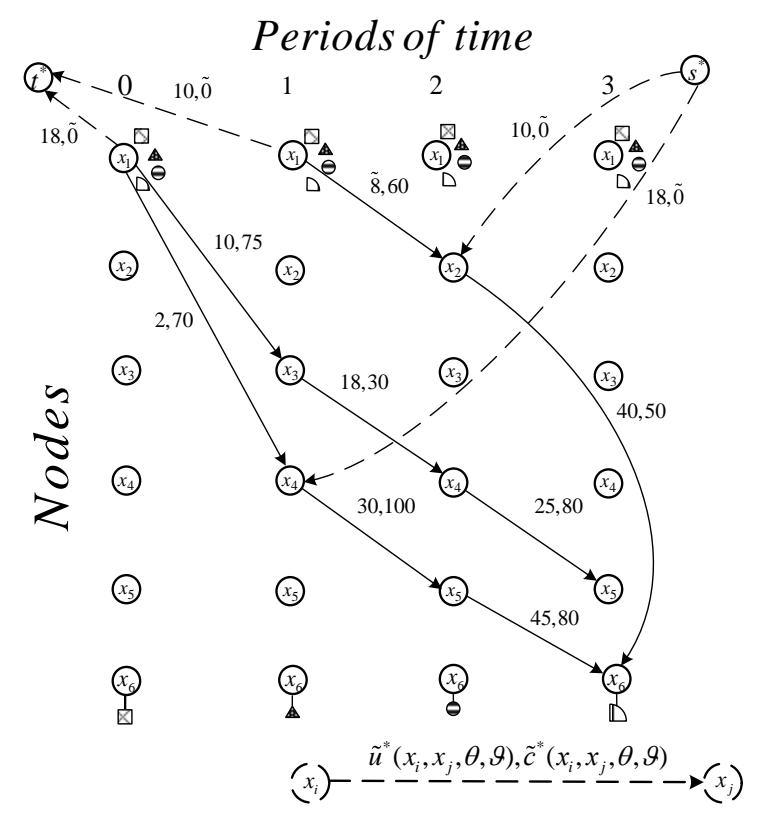

Fig. 7: Graph $\tilde{G}_{p}^{*}$ without lower flow bounds with artificial nodes and arcs.

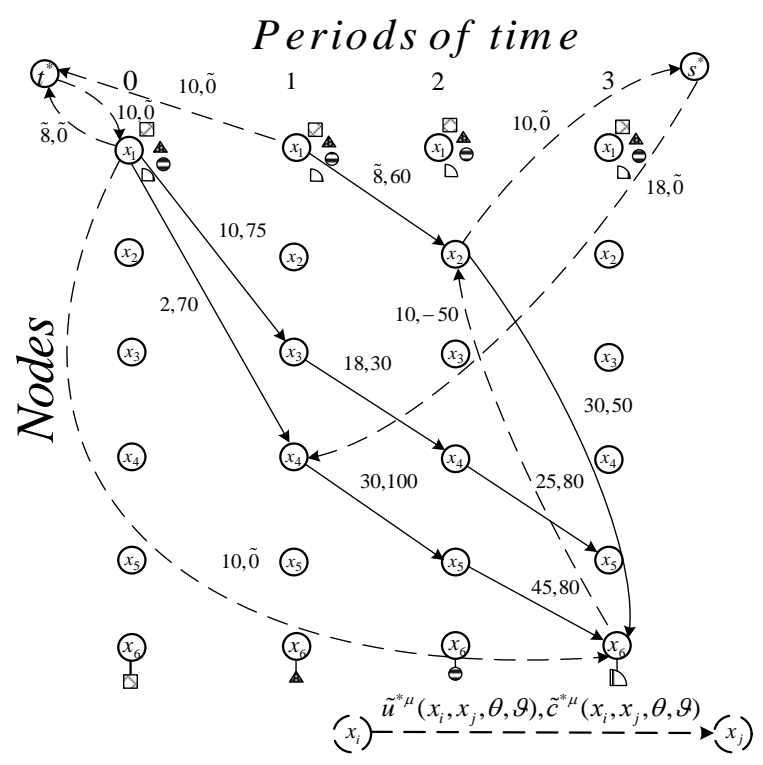

Fig. 8: Fuzzy residual network $\tilde{G}_{p}^{* \mu}$ of the graph with 10 flow units

Let us define the borders of the fuzzy flow 38 units corresponding to the maximum flow in the graph $\tilde{G}$, as shown in the Fig. 10.

The obtained result is between two basic neighboring values of the fuzzy transmission costs: 6620 with the left deviations $l_{1}^{L}=620$, right deviations $l_{1}^{R}=710$, and 7540 with the left deviations $l_{2}^{L}=690$, right devia- tions $-l_{2}^{R}=760$. According to the equation (6) $l^{L}=647, l^{R}=729$.

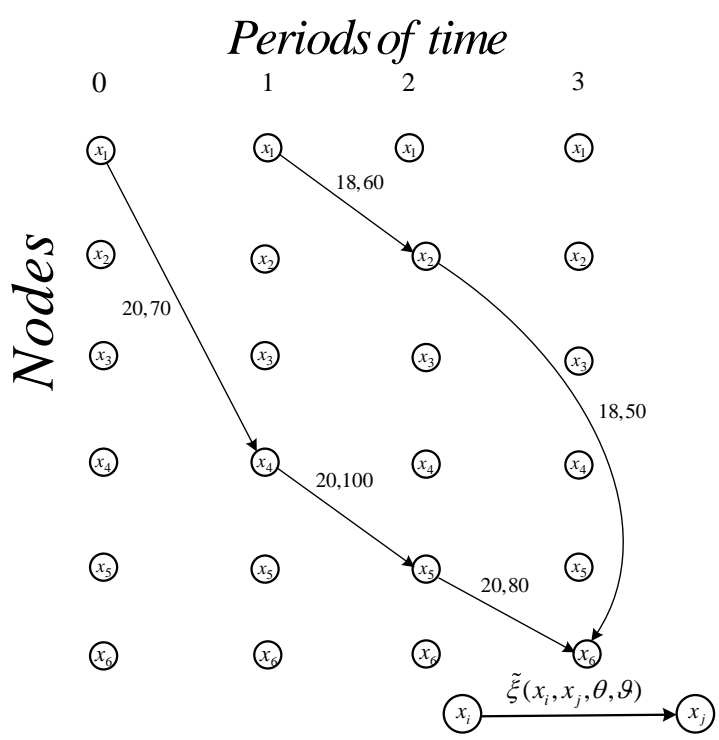

Fig. 9: Graph $\tilde{G}_{p}$ with 38 flow.

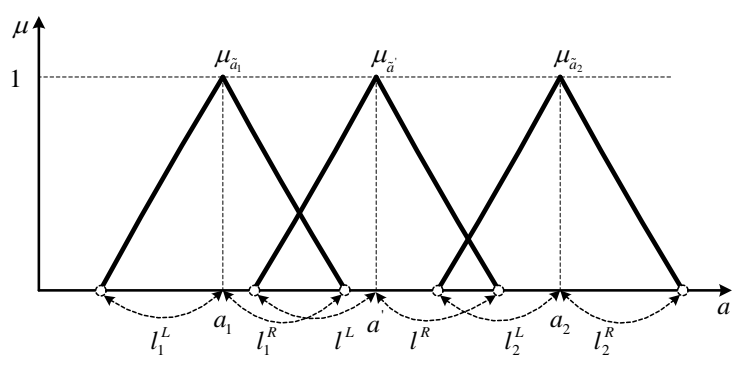

Fig 10: Defining of the membership function $\mu_{\tilde{a}}(a)$.

$$
\begin{aligned}
& l^{L}=\frac{\left(a_{2}-a^{\prime}\right)}{\left(a_{2}-a_{1}\right)} \times l_{1}^{L}+\left(1-\frac{\left(a_{2}-a^{\prime}\right)}{\left(a_{2}-a_{1}\right)}\right) \times l_{2}^{L}, \\
& l^{R}=\frac{\left(a_{2}-a^{\prime}\right)}{\left(a_{2}-a_{1}\right)} \times l_{1}^{R}+\left(1-\frac{\left(a_{2}-a^{\prime}\right)}{\left(a_{2}-a_{1}\right)}\right) \times l_{2}^{R} .
\end{aligned}
$$

The CSV-file has the same structure as presented in the Table 1. Resulting file is imported to the program, then the user selects the method of the minimum cost maximum flow finding in the fuzzy network. When the process is completed the results are available on the «Results» tab. In the tab «Iteration report» the calculated results and the values of the main variables are presented as shown in the Fig. 11. 


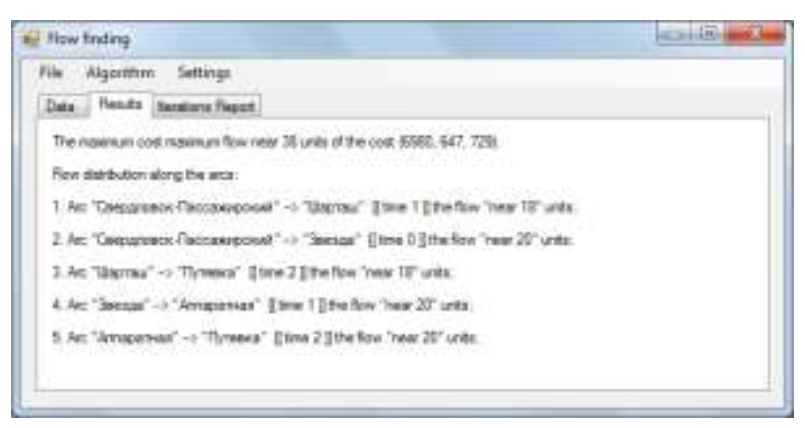

Fig. 11: Iteration report.

\section{Conclusion and future work}

The method of the minimum cost maximum flow finding in the fuzzy dynamic network is considered in this paper. The feature of the problem is in fuzzy nature of the network's parameters and possibility of the flow bounds, costs and transit times vary over time. The necessity of introducing the lower bounds is taking into account due to the peculiarity of the network. The practical implementation of the proposed method is realized by the railway map of the Russian Federation via GIS «ObjectLand».

In the future, new methods of the maximum and the minimum cost flow finding in fuzzy networks with fuzzy continuous transit times will be proposed. Moreover, new methods of the dynamic flow finding with fuzzy continuous transit times will be obtained on the networks with gains.

Acknowledgments. This work has been supported by the Russian Foundation for Basic Research, Project № 15-07-00185a.

\section{References}

[1] A. Bozhenyuk, E. Gerasimenko and I. Rozenberg, The task of minimum cost flow finding in transportation networks in fuzzy conditions. In Proceedings of the 10th International FLINS Conference on Uncertainty Modeling in Knowledge Engineering and Decision Making Word Scientific, Istanbul, pages 354359, Turkey, 26-29 August, 2012.

[2] A. Bozhenyuk and E. Gerasimenko, Flows Finding in Networks in Fuzzy Conditions. In Cengiz
Kahraman and Basar Öztaysi (Eds), Supply Chain Management Under Fuzzines, Studies in Fuzziness and Soft Computing, Springer-Verlag Berlin Heidelberg, vol. 313, Part III, pages 269-291, 2014.

[3] I. Chabini and M. Abou-Zeid, The minimum cost flow problem in capacitated dynamic networks. In Annual Meeting of the Transportation Research Board, pages 1-30, Washington, D.C., 2003.

[4] E. Nasrabadi and S.M. Hashemi, Minimum cost time-varying network flow problems, Optim. Meth. Software, vol. 25(3), pages 429-447, 2010.

[5] L.K. Fleischer and M. Skutella, Quickest flows over time, SIAM J. Comput. 36, pp. 1600-1630, 2007.

[6] X, Cai, D. Sha and C. K. Wong, Time-varying minimum cost flow problems, European J. Oper. Res. 131, pages 352-374, 2001.

[7] R.K. Ahuja, J.B. Orlin, S. Pallottino and M.G. Scutella, Dynamic shortest paths minimizing travel times and costs, Networks 41, pages 197-205, 2003.

[8] G. D. Glockner, G. L. Nemhauser and C. A. Tovey, Dynamic network flow with uncertain arc capacities: decomposition algorithm and computational results, Computational Optimization and Applications, vol. 18, pages 233-250, 2001.

[9] A. Bozhenyuk, E. Gerasimenko and I. Rozenberg, The methods of maximum flow and minimum cost flow finding in Fuzzy Network. In Ignatov, D., Kuznetsov, S., Poelmans, J. (Eds.) Concept Discovery in Unstructured Data Workshop (CDUD 2012) co-located with the 10th International Conference on Formal Concept Analysis (ICFCA 2012) May 2012, pages 1-12, Katholieke Universiteit Leuven, Leuven, Belgium, 2012.

[10] A. Bozhenyuk and E. Gerasimenko, Algorithm for Monitoring Minimum Cost in Fuzzy Dynamic Networks, Information Technology and Management Science, vol. 16 (1), pages 53-59, 2013.

[11] I. Rozenberg, C. Gittis and D. Svyatov, Geoinformation System Object Land. In Proc. IPI RAN "Systems and Means of Informatics", Science, Moscow, 2000.

[12] ObjectLand Geoinformation science [eresource]. - Acess mode: http://www.objectland.ru/. 